\title{
AN ELDERLY MAN WITH DYSPHAGIA AND DYSARTHRIA
}

Sir,

An 81-year-old man was admitted to hospital with a 2-day history of slurred speech and swallowing difficulty. There was no history of weakness in the limbs, diplopia or difficulty in breathing. He had enjoyed good health up until 
this event and his only medical problem was hypertension. He was taking losartan $50 \mathrm{mg}$, bendrofluazide $2.5 \mathrm{mg}$ and aspirin $75 \mathrm{mg}$. $\mathrm{He}$ was a smoker and consumed little alcohol occasionally.

He was well built, alert and oriented. He had good strength in all limbs and there was no focal weakness, reflexes were normal with downgoing plantars. There were no cerebellar signs. However, he was dysarthric and also had difficulty swallowing. The blood pressure on admission was 220/100 mm Hg. Full blood count, inflammatory markers and biochemical profile were within the normal range. Electrocardiogram and chest $\mathrm{x}$-ray were normal. Computed tomography (CT) scan of brain showed moderate generalised cerebral and cerebellar atrophy. With the background of hypertension and smoking in an elderly man a diagnosis of stroke was made, and the patient was transferred to stroke ward for rehabilitation. Assessment by speech and language therapist revealed reduced tongue movements with severe dysarthria, and poor swallow with no laryngeal elevation during swallow. Due to poor swallow and high risk of aspiration a Nasogastric (NG) tube was inserted for feeding. On the third day from admission the patient became short of breath and was unable to get out of bed. He had severe dysarthria and was almost unable to speak. During examination he developed bilateral ptosis, became hypoxic (pulseoximeter-saturation $70 \%$ ) and had a periarrest. A presumptive diagnosis of myasthenia gravis was made, the patient was resuscitated and pyridostigmine was administered via the NG tube. The patient showed dramatic response to pyridostigmine and was then transferred to high dependency unit for further management. His acetylcholine receptor antibodies were elevated at 388.7 units $(<4.0)$. With the diagnosis of myasthenia gravis he was commenced on pyridostigmine 60 mgs six times a day along with prednisolone $60 \mathrm{mg}$ daily. He had normal CT scan of thorax and remains well at 8 months follow-up.

Myasthenia Gravis (MG) is a potentially serious but treatable disorder of neuromuscular transmission associated with acetylcholine receptor antibodies. The hallmark of the disease is fatigable weakness, however the spectrum can range from mild ocula symptoms to severe generalised form and respiratory failure..$^{[1,2]}$ Due to vague symptoms and associated co-morbidities diagnosis of MG in the elderly can be difficult and MG can mimic stroke in elderly patients. ${ }^{\left[{ }^{[3}\right.}$ Kluin et a have described their experience of dysphagia and dysarthria in elderly patients with MG. ${ }^{[4}$ Presentation with dysphagia and dysarthria as in our patient is not a common feature of MG. Our case demonstrates that MG can be misdiagnosed as stroke in the elderly, and delay in the diagnosis can be life-threatening MG is substantially underdiagnosed in older people ${ }^{[5]}$ and should be considered as a diagnostic possibility in elderly patients with neurological features. Early and correct diagnosis may prevent complications from this treatable disease.

\section{REFERENCES}

1. Ojini FI, Danesi MA, Ogun SA. Clinical manifestations of myasthenia gravis-review of cases seen at the Lagos University Teaching
Hospital. Niger Postgrad Med J 2004;11:193-7

2. Kothari MJ. Myasthenia gravis. J Am Osteopath Assoc 2004;104:377-84

3. Kleiner-Fisman G, Kott HS. Myasthenia gravis mimicking stroke in elderly patients. Mayo Clin Proc 1998;73:1077-8

4. Kluin KJ, Bromberg MB, Feldman EL, Simmons Z. Dysphagia in elderly men with myasthenia gravis. J Neurol Sci 1996;138:49-52

5. Vincent A, Clover L, Buckley C, Grimley EJ,
Rothwell PM. UK Myasthenia Gravis Survey. Evidence of underdiagnosis of myasthenia gravis in older people. J Neurol Neurosurg Psychiatr 2003:74:1105-8

BANKAR RN, KÖHNKE A Department of Medicine, Scarborough and North East Yorkshire Healthcare NHS Trust, UK

Correspondence

RN Bankar Specialist Registrar General Hospital, (n)
Indian Journal of Medical Sciences is pleased to announce the launch of its website. The URL of the website is http://www.indianjmedsci.org.

The features of the site are:

- Free full text availability of articles in HTML as well as PDF

- Link to abstracts and full text from the cited references

- Link to PubMed abstracts of published articles by authors

- Link to related articles in PubMed

- Link from text of articles to various databases and search engines

- Facility to submit comments on articles

- Email notifications on new issue release

- Statistics of articles download and visits

- Structure based on OpenURL, DC Metadata and other international standards 